\title{
An Uncommon Liver Mass and Hypereosinophilia: Acute Fascioliasis
}

\author{
Banu Demet Ozel ${ }^{\mathrm{a}, \mathrm{b}}$, Ahmet Yagbasan ${ }^{\mathrm{a}}$, Ahmet Karaman ${ }^{\mathrm{a}}$
}

\begin{abstract}
Fascioliasis is a zoonotic disease caused by the trematode Fasciola hepatica, a liver fluke. Its usual hosts are sheep and cattle, in which it causes a disease of economic importance. Humans are accidental hosts who become infected after eating uncooked aquatic plants on which encysted organisms are present. In Turkey, clinical cases are seen in sporadic and it is difficult to recognize them; there might be delays in diagnosis and therapy. By scanning the literature, it is understood that most fascioliasis cases have been diagnosed in the biliary phase (chronic phase). Here, we presented a case of acute fascioliasis with hypereosinophilia that had mass in liver. The patient was successfully treated with triclabendazole, $10 \mathrm{mg} / \mathrm{kg} / \mathrm{day}$ for single oral dose.
\end{abstract}

Keywords: Fasciola hepatica; Hypereosinophilia; Liver mass; Triclabendazole

\section{Introduction}

Fascioliasis is a zoonotic disease caused by two foodborne trematodes, namely, F. hepatica and F. gigantica, which usually infect cattle, sheep, goats, and other domestic ruminants [1]. The World Health Organization considers fascioliasis an important human parasitic disease, with recent estimations showing that up to 17 million people are infected in worldwide. Although fasciolosis is frequently seen in developing countries, there has been an increase in the number of cases reported from developed countries in the last decade $[1,2]$.

Manuscript accepted for publication October 18, 2013

${ }^{a}$ Department of Gastroenterology, Erciyes University, Medical School, Kayseri, Turkey

${ }^{\mathrm{b}}$ Corresponding author: Banu Demet Ozel, Kikapi Mh.Hoca Ahmet

Yesevi Cd.Hidayet Eraslan Sitesi B Blok No. 12, Talas, Kayseri,

Turkey.Email: demet3032@hotmail.com

doi: http://dx.doi.org/10.4021/jmc1534w
Domesticated herbivores are the primary reservoirs of the parasite. Humans are accidental hosts, infected by ingesting contaminated water or raw aquatic vegetables, most commonly watercress [3]. Infestation with $F$. hepatica has a variable clinical presentation. In acute phase, symptoms correspond to migration of larval stage from intestine and are characterized by fever, abdominal pain, headache, pruritus, urticaria, weight loss, and eosinophilia. The chronic phase is usually asymptomatic, and the prominent sign may only be intermittent cholangitis $[4,5]$. The acute or hepatic phase of fascioliasis is difficult to diagnose. Furthermore, many physicians and even infectious diseases specialists outside countries of high endemicity are not familiar with the presentation and management of acute fascioliasis. Our report aims to improve the comprehension of acute fascioliasis by presenting and analyzing the clinical and diagnostic features of patient.

\section{Case Report}

A 60-year-old woman presented to a local hospital complaining of severe right upper quadrant pain, intermittent fever, and weakness for 4 months. On admission to the hospital her temperature was normal, and she had right upper quadrant tenderness without hepatosplenomegaly or masses with peripheral eosinophilia (40\%). An abdominal computed tomography (CT) scan with intravenous contrast demonstrated 6-7 $\mathrm{cm}$ diameter hypodense lesions with irregular limited in the right lobe of the liver (Fig. 1). An IgG enzyme immunoassay result for antibodies to Echinococcus granulosus was low positive. The patient was referred to Erciyes University School of Medicine Gastroenterology Clinic due to hypereosinophilia and liver mass with suspicion of echinococcosis.

She had been operated for a benign pancreatic cyst 2 years ago and had no prior medication. She was living in a rural area. Her family members were well. On admission, physical examination revealed right subcostal tenderness without hepatomegaly and scar of previous surgical procedure. The other physical findings were within normal. Laboratory investigation results were as follows: white blood cells (WBC) 24,900/ $\mathrm{mm}^{3}$; eosinophil 45\%; erythrocyte sedi- 


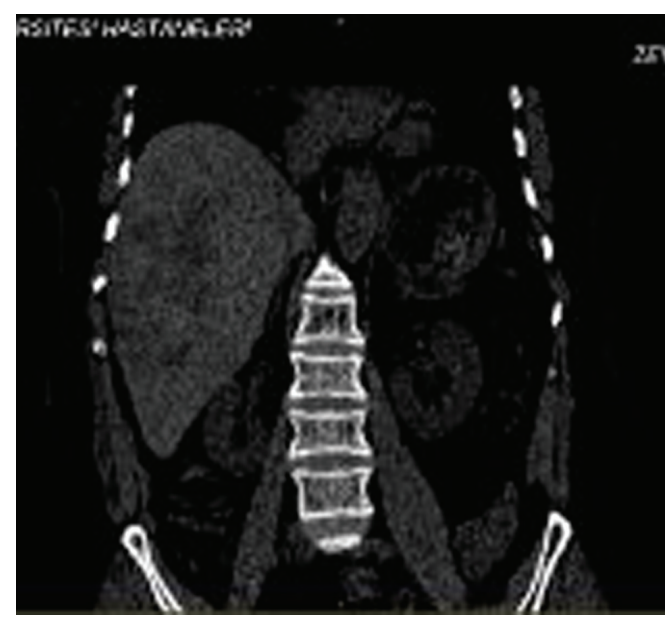

Figure 1. A 60-year-old female patient presented with right upper abdominal pain and fever lasting 16 weeks. Abdominal computerized tomographic examination showed hypodense lesions in the right lobe of the liver.

mentation rate $40 \mathrm{~min} / \mathrm{h}$; and C-reactive protein: $20 \mathrm{mg} / \mathrm{L}$; alanine aminotransferase (ALT) level was $65 \mathrm{U} / \mathrm{L}$ (normal, 5-37), aspartate aminotransferase (AST) level was $49 \mathrm{U} / \mathrm{L}$ (normal, 5-37), and alkaline phosphatase level (APL) was $266 \mathrm{U} / \mathrm{L}$ (normal, 32-155). Tumor markers including alpha fetoprotein, carcinoembryonic antigen, and carbohydrate antigen 19-9, were all within the normal range. In addition, no viral markers were detected.

Chest radiography and thorax CT were normal. Abdomen ultrasonography (US) of the patients showed hypoechoic nodular lesions which were irregular limited in the right liver lobe. However, a repeated CT scan revealed the above noted findings. In order to further assess this liver mass, the patient underwent an magnetic resonance imaging (MRI) examination. MRI showed T1 hypo, T2 hyperintens multiple nodular lesions like microabscesses with peripheral contrast enhancement (Fig. 2). A mass biopsy was performed which revealed eosinophilic infiltration at portal areas and liver parenchyma. Upper endoscopy and colonoscopy were normal. Gastric antral biopsy was normal. Bone marrow aspiration and biopsy was consistent with hypereosinophilia. Cytogenetic studies (JAK-2 mutation) were normal. Parasitological investigation of her stool was negative. Western blot for echinococcus was negative. The results of other serologic tests for amebiasis and toxocariasis were negative. The patient was questioned retrospectively and reported having eaten watercress. Therefore, Fasciola-indirect hemagglutination (IHA) test was performed and was found to be positive at a titer of 1/1,280. An endoscopic retrograde cholangiopancreatography (ERCP) was performed for visualizing the parasites in the biliary tree. Intra- and extra-hepatic bile ductus dilation and filling defect were not observed. In bile aspiration, fasciola eggs were not observed.

Acute fascioliasis was diagnosed because of hypereosin-

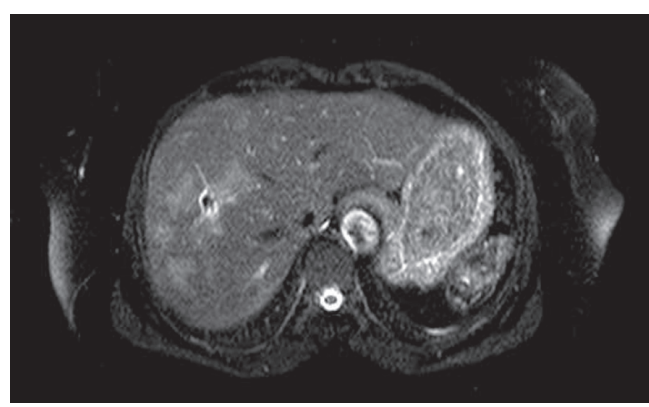

Figure 2. Abdomen MR showed multiple hypointense lesions in the right lobe of the liver.

ophilia, liver mass, positive fasciola hepatica serology, and having a history of eating watercress. The patient was treated with a two single dose $(10 \mathrm{mg} / \mathrm{kg}, 700 \mathrm{mg})$ of triclabendazole (Egaten $250 \mathrm{mg}$; Novartis, Switzerland). For follow-up study after 12 weeks, a CT scan showed almost complete resolution of the lesion.

\section{Discussion}

Fascioliasis is a rare zoonotic disease, caused by $F$. hepati$c a$, a liver fluke. F. hepatica infects sheep, cattle, and other herbivorous animals, a sheep liver fluke; humans are only accidental hosts. Most of the cases in humans have been reported from South America, Africa, Australia, Mediterranean countries, and China [6, 7]. These countries have significant sheep and livestock industries. In general, the geographic prevalence of the human disease is parallel to that of endemic animal illness. Although raising livestock is a common practice in Turkey, human fascioliasis is quite rare. In a study, the rate of seroprevalence for $F$. hepatica was $0.8 \%$ in Mersin province [8], which was a hypo-endemic level.

$F$. hepatica is a trematode, with a target location in the bile ducts. The leaf-shaped male and female adult worms reach a size of about $2 \mathrm{~cm}$ and may remain viable in the bile ducts for more than a decade. They produce eggs that are passed in feces, hatch in water, and infect a snail intermediate host. Snails release a cercarial stage of the parasite that contaminates aquatic plants ingested by sheep, cattle, or humans. When ingested, transformed metacercariae penetrate the intestine, transverse the peritoneal cavity and liver capsule, and burrow through the liver parenchyma for 1-3 months while maturing, finally entering the bile ducts to become mature adults and complete the cycle $[4,5]$.

The clinical manifestations of fascioliasis differ according to the stage of the disease. The fasciola infection has two phases: the acute (hepatic) phase and the chronic (biliary) phase. Acute infection can be associated with mild to severe illness or may be asymptomatic.

This may be related to the number of invading larvae. Two-thirds of cases are symptomatic with fever and leuko- 
cytosis. Acute fascioliasis may continue for several weeks to months and coincides with the migration of the larval flukes through the hepatic parenchyma. Hepatic enzymes may be normal or minimally raised $[8,9]$, as was observed in our patients. In general, the triad of fever, right-upperquadrant pain, and absolute peripheral blood eosinophilia should raise suspicion of hepatic fascioliasis. The acute symptoms subside soon after the larval flukes enter the bile ducts. During the second stage, the parasite resides in the biliary tract and is responsible for a variety of symptoms. Affected individuals usually complain of intermittent episodes of biliary colic, which may or may not be associated with fever and chills. Occasionally, bile duct obstruction and/or cholangitis do develop and rarely with acalculous cholecytitis, and severe hemobilia, and acute pancreatitis. Immature flukes that fail to migrate into the liver can produce ectopic masses or abscesses in many locations, most commonly appearing as subcutaneous nodules, with a surrounding eosinophilic infiltrate. In addition, a syndrome of eosinophilic pleuritis and pericarditis, without direct parasitic involvement of these structures, may accompany acute fascioliasis [10].

Repeated stool examinations may be necessary to find eggs; most often, however, they are never demonstrated [11]. In acute fascioliasis, immature worms have not initiated ovipositing; therefore, stool examination is not helpful and, as in ectopic infections, serodiagnosis is required. Serologic tests are essential to diagnose acute and chronic fascioliasis. Although these tests are quite sensitive, they may cross-react with other parasitic infections such as echinococcus and paragonimus [12]. In our case, the patient initially was referred to our gastroenterology department for treatment of hydatid cyst disease when the serologic test result was interpreted at another institution as consistent with echinococcosis. While IHA for fasciola in our patient was very high level, ecinococchus IFAT was very low (1/320) positive.

Hepatic imaging is crucial in patients with possible acute fascioliasis. A useful imaging technique is CT, which reveals hypodense focal lesions that might be confused with metastasis or abscess; more typical for acute fascioliasis are hypodense tunnel-like lesions, which, over time, slowly change in a centripetal manner. MRI, which offers no diagnostic advantage, is characterized by hypointense lesions in T1-weighted images, and hyperintense signals in T2 images. Periportal lymph nodes are not infrequent but are non-specific. The combination of upper abdominal pain, marked eosinophilia, and hypodense lesion in CT imaging is highly indicative of acute fascioliasis. Other rare causes of this combination of findings include hepatic toxocariasis and hepatic capillariasis [13]. Endoscopic retrograde ERCP is particularly effective in the biliary stage. The characteristic ERCP findings are linear, or filamentous, curved or angled, motile filling defects located in the bile duct and irregularities on the inner surface of the bile ducts $[10,14]$.

Triclabendazole is the drug of choice for this parasitosis.
Treatment consists of a single oral dose of $10 \mathrm{mg} / \mathrm{kg}$; in cases of failure, triclabendazole treatment is repeated at a higher dosage of $20 \mathrm{mg} / \mathrm{kg}$ (in two doses separated by 12-24 h). The drug is effective and has an excellent safety profile. The criteria of cure of acute fascioliasis are alleviation of clinical symptoms (sometimes a temporary exacerbation might be observed), normalization of eosinophilia, and a decrease in fasciola-specific antibodies or circulating antigen. The eosinophil count started to fall within 1 week and normalized after 8 weeks, whilst the hepatic lesion was almost completely resolved at 4 months, confirming our clinical diagnosis. Stool examinations for ova and parasites might be applied several weeks after therapy to demonstrate eradication of the parasite. Nonetheless, neither for diagnosis nor follow-up is a standardized protocol available [14]. In our patient, triclabendazole was successfully administered, and no adverse effects or recurrence were reported.

\section{Financial Support}

None.

\section{Conflict of Interest}

None.

\section{References}

1. Mas-Coma MS, Esteban JG, Bargues MD. Epidemiology of human fascioliasis: a review and proposed new classification. Bull World Health Organ. 1999;77(4):340-346.

2. Hopkins DR. Homing in on helminths. Am J Trop Med Hyg. 1992;46(6):626-634.

3. Mas-Coma S. Secondary reservoir role of domestic animals other than sheep and cattle in fascioliasis transmission in the Northern Bolivian Altiplano. Research and reviews in parasitology. 1997;57:39-46.

4. Price TA, Tuazon CU, Simon GL. Fascioliasis: case reports and review. Clin Infect Dis. 1993;17(3):426-430.

5. Noyer CM, Coyle CM, Werner C, Dupouy-Camet J, Tanowitz HB, Wiitner M. Hypereosinophilia and liver mass in an immigrant. Am J Trop Med Hyg. 2002;66(6):774-776.

6. Maclean JD, Mahanty S. Liver, lung and intestinal fluke infections. In: Guerrant, RL, Weller PF (Eds). Tropical Infectious Diseases: Principles, Pathogens and Practice, 1st ed. Philadelphia, Churchill Livingstone, 1999;1039.

7. Chen MG, Mott KE. Progress in assessment of morbidity due to Fasciola hepatica infection: a review of recent literature. Trop Dis Bull. 1990;87:R1-38.

8. Ozturhan H, Emekdas G, Sezgin O, Korkmaz M, Al- 
tintas E. Seroepidemiology of Fasciola Hepatica in Mersin province and surrounding towns and the role of family history of the Fascioliasis in the transmission of the parasite. Turk J Gastroenterol. 2009;20(3):198-203.

9. Cosme A, Ojeda E, Cilla G, Torrado J, Alzate L, Beristain $\mathrm{X}$, Orive V, et al. [Fasciola hepatica. study of a series of 37 patients]. Gastroenterol Hepatol. 2001;24(8):375380.

10. Sezgin O, Altintas E, Disibeyaz S, Saritas U, Sahin B. Hepatobiliary fascioliasis: clinical and radiologic features and endoscopic management. J Clin Gastroenterol. 2004;38(3):285-291.

11. Arjona R, Riancho JA, Aguado JM, Salesa R, GonzalezMacias J. Fascioliasis in developed countries: a review of classic and aberrant forms of the disease. Medicine (Baltimore). 1995;74(1):13-23.

12. Hassan MM, Farghaly AM, el-Gamal RL, el-Ridi AM. Cross-reactions in immunodiagnosis of patients infected with Schistosoma, Fasciola and Heterophyes using ELISA. J Egypt Soc Parasitol. 1989;19(2 Suppl):845-851.

13. Marcos LA, Tagle M, Terashima A, Bussalleu A, Ramirez C, Carrasco C, Valdez L, et al. Natural history, clinicoradiologic correlates, and response to triclabendazole in acute massive fascioliasis. Am J Trop Med Hyg. 2008;78(2):222-227.

14. World Health Organization. Report of the WHO Informal Meeting on use of triclabendazole in fascioliasis control. Geneva: WHO, 2007. 